\title{
The use of sodium polytungstate for conodont separations
}

\author{
NORMAN M. SAVAGE \\ University of Oregon, Eugene, Oregon 97403, USA
}

\begin{abstract}
Most conodont workers use heavy liquids that are carcinogenic or toxic in other ways. The use of the non-toxic water-based liquid sodium polytungstate has not been widely accepted because of reports that its high viscosity prevents the more delicate conodonts from settling, that it tends to crystallise during use, and that it is more expensive than traditional liquids. If used in the manner described below, viscosity, cystallisation and cost are no longer problems. The overwhelming advantage of safety then makes sodium polytungstate the heavy liquid of choice for conodont work.
\end{abstract}

\section{INTRODUCTION}

The number of workers engaged in conodont research has increased greatly in the past two decades so that there are now several hundred investigators and assistants engaged in processing sedimentary residues for conodonts. Most of these workers are using heavy liquids that are either carcinogenic (bromoform, tetrabromoethane) or toxic in other ways (methylene iodide). In view of the dangers involved in the use of these liquids, a search for alternatives has been underway for several years.

A likely answer to this problem is the chemical sodium polytungstate $\left(3 \mathrm{Na}_{2} \mathrm{WO}_{4} \cdot 9 \mathrm{WO}_{3} \cdot \mathrm{H}_{2} \mathrm{O}\right)$ available from Sometu, Falkenried 4, D1000, Berlin 33, West Germany, for approximately $\$ 100 / \mathrm{kg}$. A nontoxic heavy liquid can be made by the dissolution of sodium polytungstate in water. The liquid becomes highly viscous and has a tendency to crystallise toward its maximum specific gravity of 3.1 , characteristics that have led several workers to reject it as unsuitable for separating conodonts, despite the widely circulated publications by Callahan (1987) and Krukowski (1988) recommending its use. The viscosity and tendency to recrystallise are not major problems and I have found sodium polytungstate very satisfactory for conodont separation if certain procedures are adopted that result approximately in a doubling of the time needed for separating and picking compared with that needed when using tetrabromoethane. The method I have been using for the past 30 months is simple and requires equipment normally available in a conodont laboratory.

\section{PROCEDURE}

The separation of the residue is carried out in a closed separating funnel (Fig. 1 [1]) to prevent evaporation. The sodium polytungstate liquid is used at a specific gravity of 2.78 to reduce viscosity and speed settling. The mixture of liquid and residue is stirred every 30 minutes for 4 hours before the heavy fraction is released into an evaporating dish [2]. The light fraction is then run into a filter funnel [3] and filtered through a fast paper, such as a coffee filter, into a storage bottle [4]. The heavy fraction is washed several times with distilled water from a wash bottle, the washings being carefully decanted into a large 81 washings beaker [5] of distilled water. The washed heavy fraction is left to dry at room temperature and then picked for conodonts.

All the light fraction in the filter paper and the filter paper itself, together with any residue remaining in the separating funnel, on the stirring rod, and anywhere else that the heavy liquid accumulates, is washed into the large washings beaker. If it is necessary to keep the light fraction for reference or further research, it should be immersed with the filter paper in a separate smaller beaker of distilled water and stirred thoroughly before the resulting weak polytungstate solution is decanted into the washings beaker, and this should be repeated at least twice before leaving the washed light fraction to dry. I seldom keep the light fraction and so the filter paper and its contents go directly into the large beaker of washings, making recovery simpler and more efficient.

The mixture of lights and filter papers in the large beaker is stirred frequently and the level kept down by periodically decanting the clear upper part through filter [6] into washings bottle [7]. For maximum recovery of the polytungstate, the washed lights and filter papers in the large washings beaker should be washed once more with clean distilled water before being discarded. The filtered washings are poured into heat resistant Nalgene dishes [8] or very large evaporating dishes. Do not use glass beakers as these will break from the pressure of crystallisation when the solution is dried. The dishes are evaporated to dryness in a large oven [9] fitted with an exhaust fan. Three 21 dishes are used, two being kept for the initial dilute solution and a third one for the more concentrated solution that accumulates from the other two as evaporation proceeds. It is important to have a good oven and large 
dishes if the recovery of the polytungstate is to keep up with its use. When the concentrated polytungstate crystallises, distilled water is added to bring it back into solution and the specific gravity is adjusted to 2.78 in a measuring cylinder using one of the areometers [10] provided by the polytungstate supplier.

\section{DISCUSSION}

If recovered in this way, there is very little loss of polytungstate except for some that separates out as calcium tungstate and accumulates as a fine white precipitate in the bottom of the various containers. I have used $6 \mathrm{~kg}$ of sodium polytungstate to carry out over 700 separations, at a cost of less than $\$ 1$ per separation for chemicals. This is considerably less than the cost of carrying out the same work using tetrabromoethane, mainly because of the cost of acetone used in tetrabromoethane recovery. This saving on chemical cost partly offsets the increased labour costs resulting from the slower separations when using polytungstate.

Several workers have told me of their belief that polytungstate is too viscous to permit some of the very small and thin conodonts to settle. I think this problem is wholly overcome by using the liquid at a specific gravity of 2.78 , as described above. This means that rather more unwanted minerals sink than with tetrabromoethane at the common working specific gravity of 2.82. The resulting increase in picking time is a small matter when considered against the freedom from toxicity. I have carried recovery tests to see how many conodonts remain in the light fraction after using polytungstate at S.G. 2.78 and have shown this to be less than $3 \%$, which is no higher than when using tetrabromoethane. The elements that do not settle are not small delicate ones but usually specimens that have some lighter mineral particles attached to them.

There is no record of any harmful effects from using sodium polytungstate. I did my own separations using polytungstate on an open bench for 14 months and my technician has used the chemical for more than a year with the separating funnels in a fume hood but with the large washings beaker and the oven on an open bench. The manufacturer states that the chemical is non-toxic and we have no reason to disagree with him. Callahan (1987) could find no reference to the toxicity of sodium polytungstate and states that according to Kazantzis (1979), little is known about the toxicity of tungsten compounds.

When used in the way I have described, sodium polytungstate is an efficient and inexpensive alternative to organic heavy liquids for conodont separations. Most important of all, it is not known to be toxic or carcinogenic.

\section{Manuscript received October 1987 \\ Revised manuscript accepted January 1988}

\section{REFERENCES}

Austin, R. L. (Ed.) 1987. Conodonts: Investigative Techniques and Applications. Ellis Horwood Ltd. for British Micropalaeontological Society.

Callahan, J. 1987. A nontoxic heavy liquid and inexpensive filters for separation of mineral grains. Journal of Sedimentology, 57, 765-766.

Kazantzis, G. 1979. Tungsten, In Friberg et al. (Ed.), Handbook on the toxicology of metals. Elsevier, New York, 709 pp.

Krukowski, S. T. 1988. Sodium metatungstate: a new heavy mineral separation medium for the extraction of conodonts from insoluble residues. J. Paleont., 62, 314-316.
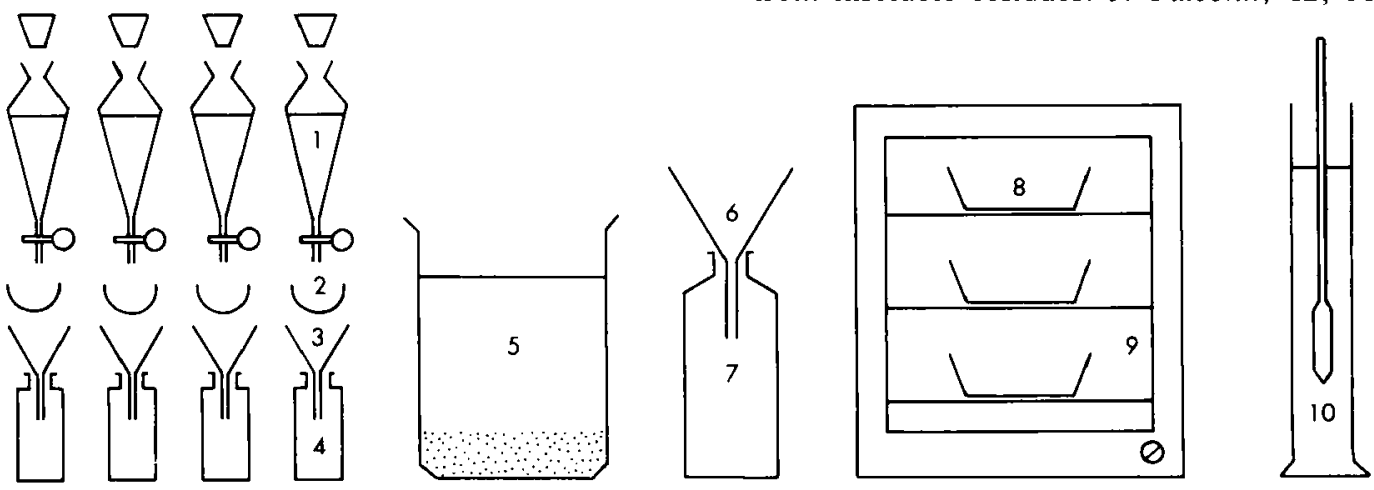

Fig. 1. Simple apparatus for conodont separation using sodium polytungstate. The numbered items referred to in the text are: 1, Separation funnels with stoppers; 2 , Small dishes for collecting the heavy fraction with conodonts; 3, Funnels and filter papers for recovering sodium polytungstate; 4, Polyethylene containers for sodium polytungstate; 5 , Large 81 beaker with distilled water into which are placed filter papers with light fraction, and other items from which sodium polytungstate needs to be recovered; 6, Large funnel and filter paper for filtering washings from 5; 7, large bottle to collect filtered washings; 8 , Nalgene heat resistant dishes for evaporating washings to dryness; 9, Large oven with exhaust fan; 10, Measuring cylinder and areometer for adjusting the specific gravity of recovered polytungstate. 